\title{
Effects of a Simultaneous Bombardment with Ions of Low Energy on the Growth and Microstructure of $\mathrm{Ag} / \mathrm{Ni}$ Multilayers
}

\author{
C. Minault ${ }^{1}$, C. Jaouen, F. Tamisier, J. Allain and Ph. Guérin \\ Laboratoire de Métallurgie Physique, UA 131 du CNRS, Université de Poitiers, SP2MI, BP. 179, \\ 86960 Futuroscope cedex, France
}

\begin{abstract}
The effect of argon ion bombardment on the structure and properties of nickel and silver films was studied as a function of the energy in a very low energy range $0-300 \mathrm{eV}$. The effect of ion irradiation on the structure of the nickel films appears very different at high energy $(\mathrm{E}>100 \mathrm{eV})$ as opposed to $25-75 \mathrm{eV}$. The microstructure looks to result from an interplay between different mechanisms related to surface and bulk damage varying across the energy range. The first results of a study concerned with the elaboration and characterization of $\mathrm{Ag} / \mathrm{Ni}$ multilayers (composition modulation $\Lambda=82 \AA$ ) deposited under Ar-ion irradiation are reported and compared with those obtained by Ion Beam Sputtering without ion assistance. X-ray diffraction scans show that coherent multilayers are obtained in both cases. The analysis of the profiles shows that the structural intralayer parameters are unchanged. On the other hand, a strong enhancement of the texture under ion irradiation is clearly shown but with a substantial decrease of the coherency length.
\end{abstract}

\section{INTRODUCTION}

Metallic multilayers can be fabricated using a variety of deposition techniques such as Thermal Evaporation, Molecular Beam Epitaxy and Sputtering. In the latter and with the majority of the ion sources, the average energy of the sputtered atoms typically ranges from 10 to $40 \mathrm{eV}$. The microstructure of films deposited with this technique is noticeably different to that of films deposited using Thermal Evaporation. These differences may be accounted for by assistance effects, due to this relatively high energy of the depositing atoms. In the last decade, this technique has also appeared very attractive to produce epitaxial metallic multilayers with a high degree of quality and sharp interfaces. An other way of increasing the surface mobility and inducing structural rearrangements of the growing film is to use a second ion beam directed towards the substrate. This technique, known as dual ion-beam sputtering (DIBS), presents the advantage of allowing the flux, energy and angle of incidence of the irradiation to be controlled independently from the atomic deposition [1]. Apart from two studies concerning the synthesis of coherent $\mathrm{Mo} / \mathrm{Fe}$ [2] and $\mathrm{Fe} / \mathrm{Ni}$ multilayers [3], little research has been carried out into the irradiation effect of a low energy ion beam incident on the substrate during the growth of multilayers.

In this paper, we report a systematic study of the effects of the ion energy on the structure of elemental $\mathrm{Ag}$ and Ni thin films. Then, we present the first results of a study concerned with the fabrication and characterization of $\mathrm{Ag} / \mathrm{Ni}$ multilayers deposited with this DIBS system, and compare these results with those obtained by Ion Beam Sputtering without assistance. This superlattice system is particularly interesting because of the complete immiscibility of $\mathrm{Ag}$ and $\mathrm{Ni}$ and of the high lattice mismatch $(\approx 15 \%)$. We show that assistance favours the stacking of the atomic planes in the $<111>$ direction resulting in an increased film coherence without increase of intermixing.

\section{EXPERIMENTAL}

$\mathrm{Ag}$ and $\mathrm{Ni}$ thin films and $\mathrm{Ag} / \mathrm{Ni}$ multilayers were prepared by ion beam sputtering in a computer-controlled ion-beam dual ion-beam chamber. This system uses an argon primary ion beam generated first from a RF source and then directed at the target by an accelerating voltage. A second defocused ion-beam source, directed towards the substrates, is used for a low-energy (20-500 eV) irradiation during deposition, allowing ion-beam assisted deposition to take place. Target and substrate holders, tilted $45^{\circ}$ away from the primary and secondary beams, are parallel and face each other. The argon ion energy and beam current from the sputter gun were $1.2 \mathrm{keV}$ and $80 \mathrm{~mA}$, respectively. The argon ion energy from the secondary gun was varied in the range $25-500 \mathrm{eV}$ while the beam current was kept fixed at the minimal operating

1 Present address: J.J. Thomson Physical Laboratory, University of Reading, Reading, Berkshire, U.K. 
value of $20 \mathrm{~mA}$, yielding an ion flux of $510^{14} \mathrm{Ar} \mathrm{cm}^{-2} \mathrm{~s}^{-1}$. The base pressure was typically $210^{-8}$ Torr prior to deposition. The operating argon pressure during the deposition is $10^{-4}$ Torr. High-purity targets and Ar gas were used. Monocrystalline Si wafers, mounted on a rotating substrate-holder, were used as substrates. Layer thicknesses of pure films were separately measured by small angle X-ray diffraction. A calibration of the individual deposition rates was thus obtained. Deposition rates for $\mathrm{Ag}$ and $\mathrm{Ni}$, without the secondary ion beam, were approximately 1.8 and $0.8 \AA / s$, respectively. From these figures, ion flux to atom flux ratios I/A of 0.36 and 0.67 were found for $\mathrm{Ag}$ and $\mathrm{Ni}$ under ion beam assistance. Multilayers (Ag/Ni) 15 were fabricated by successive deposition of silver and nickel with a composition modulation of $80 \AA$. Relative thicknesses of the layers were adjusted in order to obtain the equiatomic composition. X-ray diffraction measurements were carried out using a conventional diffractometer using the $\mathrm{Cu}-\mathrm{K} \alpha$ radiation in the $\theta-2 \theta$ reflection geometry. Samples were examined in both "symmetric" and "asymmetric" (large angle rocking curves) modes in order to determine the superlattice period, the interface quality, lattice structure of the individual layers. Composition of the $\mathrm{Ag} / \mathrm{Ni}$ multilayers was determined by EDX analysis and compared to the nominal composition.

\section{RESULTS AND DISCUSSION}

\subsection{Effects of a low-energy ion irradiation on the growth of $\mathrm{Ag}$ and $\mathrm{Ni}$ thin films}

The effects of a low-energy irradiation on the growth of elemental $\mathrm{Ag}$ and $\mathrm{Ni}$ films were investigated in the $0-300 \mathrm{eV}$ range using Ar ions.

\subsubsection{Characterization}

3.1.1.1 Surface erosion When ion assistance is carried out during growth, the resputtering effect must be taken into account. In order to do this, $\mathrm{Ag}$ and $\mathrm{Ni}$ thin films were grown under ion beam assistance for a constant deposition time. Total thicknesses of the films were deduced from simulations of the x-ray reflectometry spectra and compared to that $t$ of a film deposited for the same time without ion beam assistance. Figure 1 displays the variation of the relative surface erosion, defined as the relative thickness decrease $\Delta t / t$ due to sputtering during deposition under ion beam assistance, as a function of the energy of the incident particles.

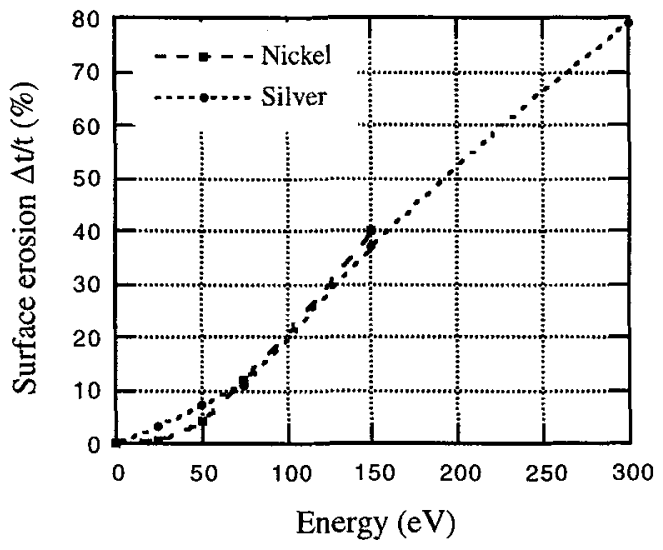

Figure 1: Dependence of the surface erosion $\Delta \mathrm{t} / \mathrm{t}$ with the $\mathrm{Ar}$ ion-energy

Due to the choice of constant ion fluxes for both primary and assistance beams, similar evolutions of $\Delta t / t$ are observed for nickel and silver depositions. Nevertheless, the corresponding sputtering yield $Y$, defined as the number of sputtered atoms by incident ion is strongly depending on the characteristics of the 
material. For a given Ar ion-fluence $\phi$, Y may be evaluated using the relation $\mathrm{Y}=\mathrm{n} \Delta \mathrm{t} / \phi$, where $\mathrm{n}$ is the atomic layer density and $\Delta t$ the measured thickness decrease. So, values of 0.19 and 1.04 can be deduced for silver films while values of 0.06 and 0.59 are found for nickel ones, those two values corresponding in each case to $\mathrm{Ar}$-ion energy of $50 \mathrm{eV}$ and $150 \mathrm{eV}$, respectively. One can stress that the sputtering rate remains rather weak below $50 \mathrm{eV}$, then increases quasi-linearly with the ion energy.

Thus, a critical value exists below which the sputtering effects are considerably reduced. This may testify that the corresponding average recoil energies are in that case inferior to the threshold value required to eject an atom from the surface. Moreover, these results show that above this threshold, for the incident ratio of our experiments, sputtering effects are significant since value of the surface erosion as high as $20 \%$ is reached for Ar-ion energy of only $100 \mathrm{eV}$.

The microstructure of silver and nickel thin films was mainly investigated using $x$-ray diffraction experiments. Fig $2 \mathrm{a}, \mathrm{b}$ displays the X-ray diffraction scans obtained in the $\theta$-range corresponding to the (111) and (200) reflexions. Various physical properties can be deduced from the analysis of these spectra such as the lattice parameter, the crystalline size and the crystallographic orientation.
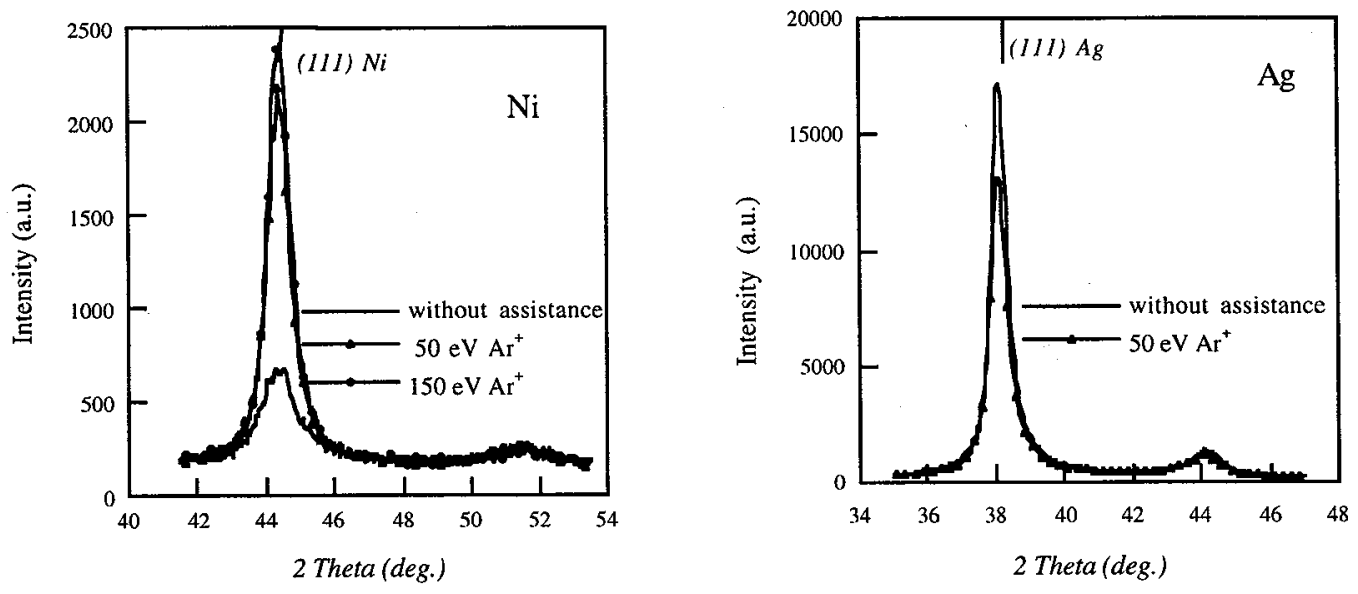

Figure 2: X-ray diffraction spectra obtained from sputtered nickel and silver thin films deposited without and with Ar-ion assistance.

3.1.1.2 Film orientation Though both our silver and nickel deposited films present a $\langle 111\rangle$ texture often observed for evaporated face-centered-cubic (fcc) metals, their evolution under irradiation differs noticeably. For nickel films, a strong increase of the relative intensity of the (111) reflexion with the ion energy is clearly observed Fig. 2a. By contrast, for silver films, no clear effect of the ion irradiation can be seen, which is why we only show the $x$-ray scan obtained with Ar-energy of $50 \mathrm{eV}$ (Fig. 3b). In the present study, the degree of the preferential (111) orientation is characterized by the $(111) /(200)$ peak intensity ratio compared to its value for random orientation in fcc polycrystalline films. Fig 3.a shows the evolution of this orientation coefficient as a function of the ion energy. The behaviour for nickel films is complex. The degree of orientation increases strongly over the $0-100 \mathrm{eV}$, then decreases at higher energies. The rocking curves across the (111) peaks of the film give information on the mosaic distribution of the epitaxial film as well as the in-plane crystalline quality of the layers. Fitting the data points with a Gaussian curve, we deduced full widths at half maximum FWHM. Very similar values are obtained from the (222) peaks which suggest that the values of FWHM are predominantly due to misorientations of the growth axis. The results are plotted Fig.3b as a function of the energy of the Ar ion assistance-beam for nickel and silver films. Finally, we can stress the good correlation between the evolution of the wide of the rocking curve (Fig. 3b) with the preferential orientation, as seen from the relative intensity of the (111) reflexion peak (Fig. 3a). 
3.1.1.3 Crystallite size The average crystallite size of the oriented (111) grains of the Ni and Ag films are determined from peak broadening of the $x$-ray (111) peak using the Scherrer formula. Correction for the instrumental broadening has been made. Due to the specular conditions used in the experimental selected geometry, the crystallite size is determined in the normal direction to the film surface. The results are plotted (Fig. 3c) as a function of the incident energy of the bombarding Ar particles. A relative good correlation between grain growth and preferential orientation can be noted for the nickel films: the grain size is larger in more oriented-(111) films or, inversely, smaller for more random orientation.

3.1.1.4 Interplanar spacing The lattice d-spacings for (111) and (200) oriented grains were measured from each diffraction pattern. Values of 2.361 and $2.053 \AA$, respectively, were deduced for the silver films, somewhat higher than bulk ones (2.359 and 2.044A). Surprisingly, no significant variation with the ion energy was observed. Similar results were observed for all nickel films. A clear increase of the d-spacings is observed for both (111) and (200) oriented grains since values of 2.043 and $1.780 \AA$ (bulk ones: 2.034 and $1.762 \AA$ ) were determined whatever the deposition conditions. Because of the Bragg-Brentano geometry used, the observed increases in lattice spacings are normal to the film surface and such lattice strains may be attributed to important biaxial compressive stresses in the planes of the films.
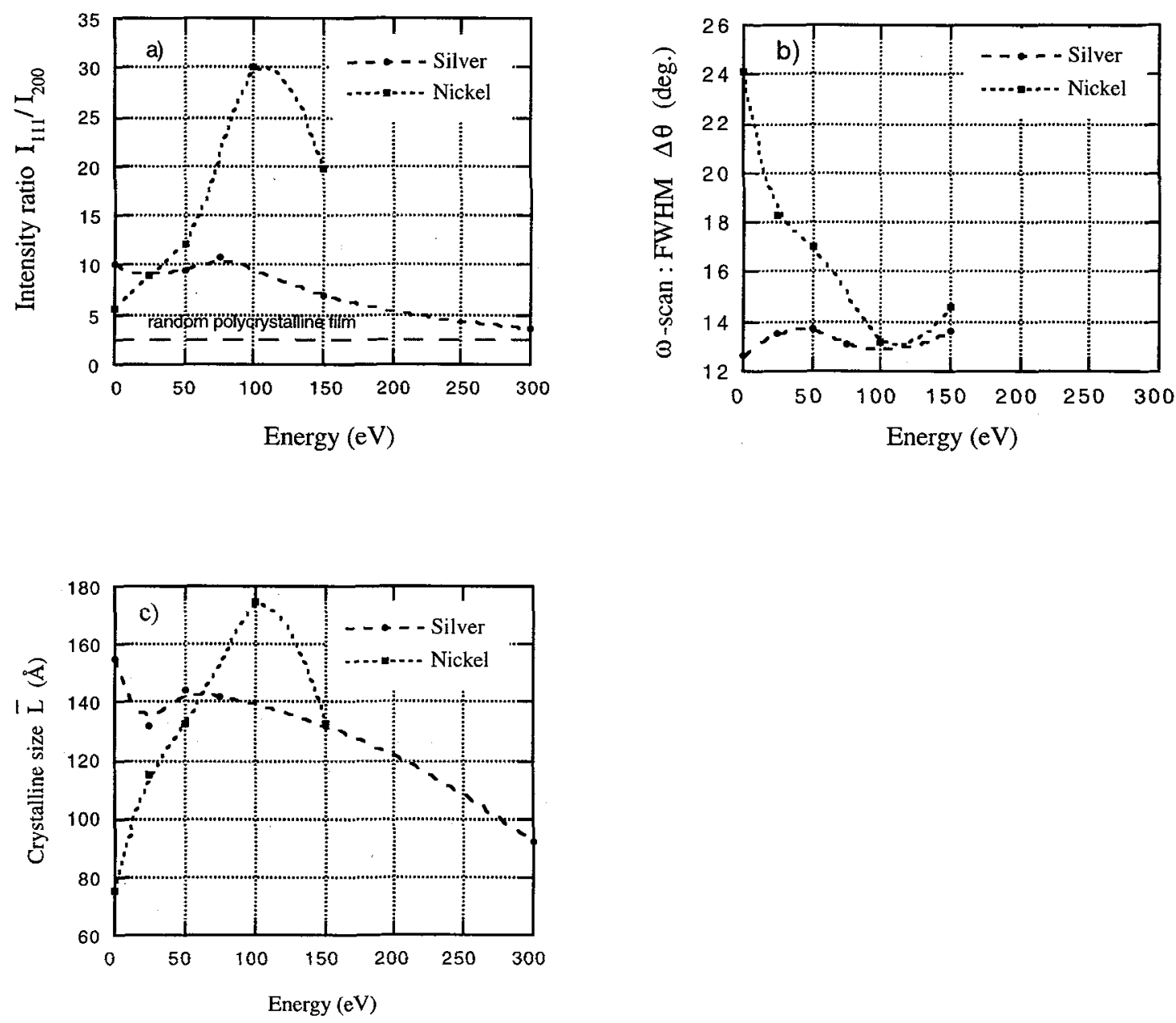

Figure 3: Evolution of different structural parameters as a function of the energy of Ar-ions for silver and nickel films a) Relative $(111) /(200)$ x-ray reflexion peak intensity; b) Full width at half maximum FWHM of the rocking curve; c) Average crystalline size $\overline{\mathrm{L}}$ 


\section{1.2 Discussion}

The above results show, in general accordance with the literature, that low energy ion irradiation during growth is able to induce deep structural changes in the nickel films. It is clear, from the trends outlined above that the (111) crystalline orientation, is favoured by the ion irradiation. In fcc lattices, the (111) planes are the most densely packed. Such planes present the lowest surface energy and their growth is thermodynamically favoured. Ion irradiation, by increasing surface mobility and densification of the surface layers may promote this process. Nevertheless, the observed behaviour as a function of the ion energy suggests an interplay of different mechanisms with antagonist effects. One can speculate that surface diffusion and forward sputter densification would be more dominant at low energy and then outplayed by bulk damage such as the disorder caused by the collision cascade and sputtering at higher energies. It is difficult in this regard to understand why the same behavior is not observed in silver films. One can additionally assume that intrinsic characteristics of the materials, such as the Debye temperature, are also determinant. Thus, we can think that the energy needed for surface diffusion of the silver atoms is relatively low and would be on average transmitted to the atoms by direct sputtering.

Finally, the present study suggests that ion assistance may mediate the (111) preferential growth of the nickel films in a relatively narrow Ar-ion energy range $(25-100 \mathrm{eV})$, in which surface displacements would be favoured while sputtering and bulk damage would be avoided or reduced. If one refers to the analytical calculations of Tsao et al [4], the existence of such an energy window would be the result of the difference in the threshold energies for surface and bulk displacements. That is why, in this preliminary study, we selected an intermediate energy of $50 \mathrm{eV}$ to study the role of the ion assistance on the structural properties of $\mathrm{Ag} / \mathrm{Ni}$ multilayers.

\subsection{Synthesis and structural characteristics of $\mathrm{Ag} / \mathrm{Ni}$ multilayers}

The role of a low-energy ion irradiation on the growth of $\mathrm{Ag} / \mathrm{Ni}$ multilayers was examined. Taking account the above results, it appears that the ion beam energy of $50 \mathrm{eV}$ yields advantageous effects (texture, grain size), mainly for nickel films, with no important visible damage. Deposition times were adjusted under ion assistance, taking account the sputtering rates deduced from the previous experiments, in order to synthetize multilayers with similar compositional modulation.

Figure 4 shows the $\theta-2 \theta$ scans performed about the (111) Bragg reflexions of the two series of $\mathrm{Ag} / \mathrm{Ni}$ multilayers produced without ion assistance and under $50 \mathrm{eV}$ Ar ion irradiation.

In agreement with the previous $\mathrm{X}$-ray investigation of sputtered $\mathrm{Ag} / \mathrm{Ni}$ superlattices by Rodmacq [5], these superlattices are polycrystalline (TEM observations), with a well defined (111) texture perpendicular to the layers.

The $x$-ray profiles, as far as peak positions and relative intensities are concerned, are remarkably similar. Thus, the main structural parameters of the two superlattices (lattice spacings in the elemental layers $\mathrm{d}_{\mathrm{Ag}}$, $\mathrm{d}_{\mathrm{Ni}}$, the number of atomic planes per layer $\mathrm{n}_{\mathrm{Ag}}, \mathrm{n}_{\mathrm{Ni}}$ and the interdiffused thickness at the interfaces $\sigma_{\text {int }}$ ) are very close. However, pronounced changes are also seen. First, a strong increase (by a factor of 1.4) of the total scattered intensity $l$, in the $2 \theta$-range corresponding to the (111) reflexions of the $\mathrm{Ag}$ and Ni films, is clearly shown. This indicates an improvement of the (111) stacking. Furthermore, a very sensitive reduction of the mosaic distribution, as deduced from the rocking curves performed on the angular position of the "- 2 " superlattice peak (peak located at $2 \theta \cong 38^{\circ}$ ), confirms this trend. By fitting the data with a Gaussian curve, we obtain full widths at half maximum of $14^{\circ}$ and $12^{\circ}$ for the $\mathrm{Ag} / \mathrm{Ni}$ multilayers deposited without and with assistance, respectively. Second, and surprisingly, an increase of the width of the satellite peaks is also seen, which displays a loss of coherency. Hence, the simple analysis of the full-width at half maximum indicates that the characteristic coherency length $\zeta$ decreases by a factor approximately of $\approx 1.5$, varying from 220 to $140 \AA$.

However, a complete determination of the structural parameters including interfacial parameters requires modelling of the superlattice structure to compare calculated profiles with measured ones. By adjusting the parameters of the model to fit the diffraction spectrum, it is possible to obtain the structure of the constituent layers. In order to do this, we used the program SLERF, written by Gladyszewski and coworkers [6], based on a Monte Carlo Method. This program assumes that the superlattice consists of a distribution of well-orientated grains, smaller than the total multilayer thickness, with an average size $\mathrm{D}_{\mathrm{g}}$ and related fluctuation sig $\mathrm{D}_{\mathrm{g}}$. Besides the main parameters above enumerated, roughness of the layers are also simulated introducing discrete fluctuations of the elemental layer thicknesses: $\operatorname{sig} \mathrm{n}_{\mathrm{Ag}}$, sig $\mathrm{n}_{\mathrm{Ni}}$. Such an approach is well adapted to describe superlattices obtained by the sputtering method. The best fits to the experimental profiles are shown in Fig. 4 and the various parameters are given in Table 1. 


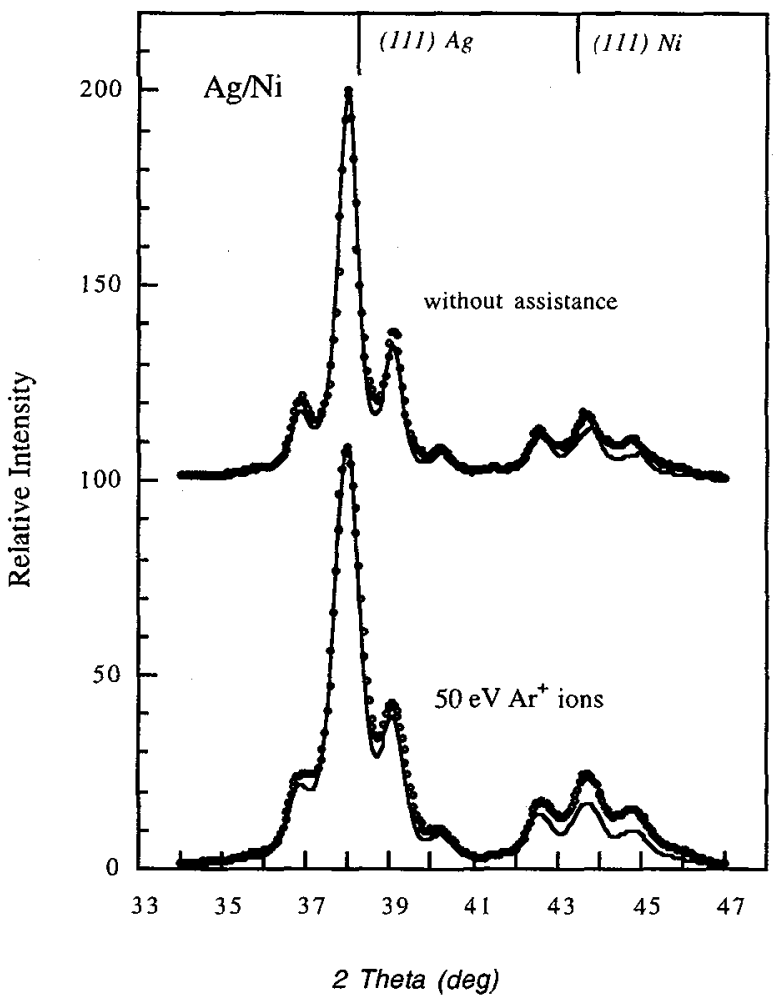

Figure 4: Experimental (points) and calculated (full curves) x-ray $\theta-2 \theta$ spectra of $\mathrm{Ag} / \mathrm{Ni}$ multilayers obtained a) without ion assistance; b) under $50 \mathrm{eV}$ Ar ion-irradiation

Table 1 Structural parameters (cf. text) of the $[\mathrm{Ag}(52 \AA) / \mathrm{Ni}(30 \AA) \times 15]$ superlattices, obtained without ion assistance and ionbeam assisted by $50 \mathrm{eV} \mathrm{Ar}^{+}$, as deduced from the simulations by the SLERF code [6].

\begin{tabular}{|c|c|c|}
\hline Parameter & $\begin{array}{c}\mathrm{Ag} / \mathrm{Ni} \\
\text { without ion-assistance }\end{array}$ & $\begin{array}{c}\mathrm{Ag} / \mathrm{Ni} \\
\text { ion-assisted by } 50 \mathrm{eV} \mathrm{Ar}^{+}\end{array}$ \\
\hline $\begin{array}{l}\mathrm{d}_{\mathrm{Ag}}(\AA) \\
\mathrm{d}_{\mathrm{Ni}}(\AA) \\
\mathrm{n}_{\mathrm{Ag}}(\mathrm{ML}) \\
\mathrm{n}_{\mathrm{Ni}}(\mathrm{ML}) \\
\text { sig } \mathrm{n}_{\mathrm{Ag}}(\mathrm{ML}) \\
\operatorname{sig} \mathrm{n}_{\mathrm{Ni}}(\mathrm{ML}) \\
\mathrm{D}_{\mathrm{g}}(\AA) \\
\operatorname{sig} \mathrm{D}_{\mathrm{g}}(\AA)\end{array}$ & $\begin{array}{l}2.366 \\
2.048 \\
21.9 \\
14.6 \\
1.1 \\
0.7 \\
51 \\
76\end{array}$ & $\begin{array}{l}2.366 \\
2.048 \\
21.9 \\
14.6 \\
1.1 \\
0.7 \\
54 \\
56\end{array}$ \\
\hline$\sigma_{\text {int }}(\mathrm{ML})$ & 0.5 & 0 \\
\hline$\Lambda(\AA)$ & 81.7 & 81.7 \\
\hline
\end{tabular}


One can notice that the ion assistance does not modify the values of the interplanar distances. In both cases, as often observed in superlattices produced by sputtering [7], the interplanar distances are slightly larger than those for the bulk materials. The average numbers of monolayers as well as the superlattice period are very close. The roughness of $\mathrm{Ni}$ and $\mathrm{Ag}$ layers, as characterized by $\operatorname{sig} \mathrm{n}_{\mathrm{Ag}}$ and sig $\mathrm{n}_{\mathrm{Ni}}$, are also unchanged, in good agreement with the x-ray reflectometry measurements (not presented here). The variation of the interdiffused layer is only equal to the uncertainty. Thus, all the main structural parameters remain unchanged. In this modeling scheme, the only differences concern the parameters related to the coherent grain size distribution. The strong broadening of the satellite peaks observed under ion assistance could be mainly explained by a reduction of the width of the grain size distribution. However, we must emphasize that the variations of such statistical parameters call for caution in the interpretation of the results. On the other hand, the fact that the average grain size remains rather unchanged, while the (111) crystalline orientation is considerably increased, appears unrealistic. Other parameters that are not included in this model could explain the observed differences in the broadening of the superlattice peaks. We think in particular to a fluctuation in the interfacial spacing. In such a lattice-mismatched system, the lattice positions at the interfaces are probably not well defined and the simulation of such a continuous disorder causes also a broadening of the peaks [8]. This would appear to be a more convincing hypothesis since it has already been shown that codeposition of silver and nickel can lead to the achievement of an amorphous phase [9$10]$. However, complementary analysis is required to validate this idea.

In conclusion, this study shows that Ar-ion beam assistance at very low energy yields an important enhancement of the texture of $\mathrm{Ag} / \mathrm{Ni}$ multilayers without inducing some significant variation of the main structural layer parameters. On the other hand, the observed broadening of the satellite peaks cannot be interpreted in the framework of the modelling scheme but it is suggested that this effect could be related to an atomic position disorder introduced at the interfaces. Finally, we must emphasize that additional studies, varying ion energy and ion mass, are required to obtain a good understanding of the effects of an ion-beam assistance on the multilayer microstructure.

\section{Acknowledgements.}

The authors would like to thank G. Gladyszewski and coworkers for the use of their x-ray refinement program.

\section{References}

[1] See for example: Roy R.A., Cuomo J.J. and Yee D.S., J, Vac. Sci. Technol. A6 (1988) 1621-26; Roy R., Surface and Coatings, 51 (1992) 3203-11.

[2] Window B. and Sharples F., J. Mater. Res., 3 (1988) 856-61.

[3] Kim C., S.B. Qadri, H.Y. Yu, K.H. Kim and A.S. Edelstein, J. Vac. Sci. Technol. A, 8 (1990) $1407-10$.

[4] Tsao J.Y., Chason E., Horn K.M., Brice D.K. and Picraux S.T., Nucl. Instr. and Meth. B, 39 (1989) $72-77$.

[5] Rodmacq B., J. Appl. Phys., 70 (1991) 4194-201.

[6] Gladyszewski G. and P. Mikolajczak, Appl. Phys., A48 (1989) 521;Gladyzewski G., Thin Solid Films, 204 (1991) 473.

[7] Pacaud J., Gladyszewski G., Jaouen C., Naudon A., Goudeau Ph. and Grilhé J., J. Appl. Phys., 73 (1993) 28.

[8] Locquet J.P., Neerinck D, Stockman L., Bruynesaraede Y and Schuller I.K., Phys. Rev. B, 38 (1988), 3572.

[9] Hauser .J.J., Phys. Rev. B, 12 (1975) 5160-5.

[10 Munoz A., Miranda H., Cumbrera F.L., Conde A. and Marquez R., Thin Solid Films, 88 (1982) 211 17. 